Commun. Korean Math. Soc. 29 (2014), No. 3, pp. 387-399

http://dx.doi.org/10.4134/CKMS.2014.29.3.387

\title{
FIBONACCI NUMBERS AND SEMISIMPLE CONTINUED FRACTION
}

\author{
Eunmi Choi
}

ABstract. The ratios of any two Fibonacci numbers are expressed by means of semisimple continued fraction.

\section{Introduction}

The Fibonacci sequence $\left\{F_{n}\right\}_{n \geq 1}$ is a series of numbers that begins with $F_{1}=F_{2}=1$ and each next is the sum of the previous two terms. The Lucas sequence $\left\{L_{n}\right\}_{n \geq 1}$ is a modified Fibonacci sequence starting from $L_{1}=1$ and $L_{2}=3$. A number of properties of these sequences were studied by many researchers. Among them, the ratios $\frac{F_{n}}{F_{n-1}}$ and $\frac{L_{n}}{L_{n-1}}$ of two successive terms of each sequence were investigated by means of simple continued fraction ([2], [3], [4] and [6]).

This work is devoted to studying the ratio $\frac{F_{n}}{F_{k}}$ for any $n$ and $k$. For the purpose, a semisimple continued fraction will be defined and compared to a simple continued fractions. We shall show that $\frac{F_{n}}{F_{k}}$ is expressed by a semisimple continued fraction more efficiently than by a simple continued fraction. And it will be seen that semisimple continued fraction may yield any large Fibonacci numbers, like $F_{105}$ a 22 digit number.

\section{Semisimple continued fractions}

We begin with a lemma that provides a motivation of this work.

Lemma $2.1([5])$. Let $k, t$ and $r$ be positive integers. If $t \geq 2$ and $r \leq k$, then we have the following relations.

(1) $L_{k t+r}=L_{k} L_{k(t-1)+r}+(-1)^{k-1} L_{k(t-2)+r}$, so $L_{n}(n=k t+r)$ is expressed by only three Lucas numbers $L_{k}, L_{r}$ and $L_{k+r}$.

(2) $F_{k t+r}=L_{k} F_{k(t-1)+r}+(-1)^{k-1} F_{k(t-2)+r}$, so $F_{n}(n=k t+r)$ is expressed by $L_{k}, F_{r}$ and $F_{k+r}$.

Received February 11, 2014; Revised June 19, 2014.

2010 Mathematics Subject Classification. Primary 11B37, 11 B39.

Key words and phrases. Fibonacci sequence, Lucas sequence, continued fraction. 
Note that $r$ may be considered as the remainder of $n$ when divided by $k$, but we assume $1 \leq r \leq k$ because the sequences start from $F_{1}=L_{1}=1$.

If $k=5$, then $L_{17}=L_{5} L_{12}+L_{7}$ and $L_{12}=L_{5} L_{7}+L_{2}$, so

$$
\frac{L_{17}}{L_{12}}=\frac{L_{5} L_{12}+L_{7}}{L_{12}}=L_{5}+\frac{1}{\frac{L_{12}}{L_{7}}}=L_{5}+\frac{1}{\frac{L_{5} L_{7}+L_{2}}{L_{7}}}=L_{5}+\frac{1}{L_{5}+\frac{1}{\frac{L_{7}}{L_{2}}}}
$$

and again

$$
\frac{L_{22}}{L_{17}}=\frac{L_{5} L_{17}+L_{12}}{L_{17}}=L_{5}+\frac{1}{\frac{L_{17}}{L_{12}}}=L_{5}+\frac{1}{L_{5}+\frac{1}{L_{5}+\frac{1}{\frac{L_{7}}{L_{2}}}}} .
$$

These fractions yield a motivation to define a sort of continued fraction composed of only Lucas numbers. Let $n \geq 2$. For real numbers $a_{0}, b_{0} \geq 0$ and $a_{i}>0, b_{i}>1(i=1, \ldots, n)$, the fractions

$$
a_{0}+\frac{1}{a_{1}+\frac{1}{\ddots \cdot a_{n-2}+\frac{1}{\frac{a_{n-1}}{a_{n}}}}} \text { and } b_{0}-\frac{1}{b_{1}-\frac{1}{\ddots b_{n-2}-\frac{1}{\frac{b_{n-1}}{b_{n}}}}}
$$

are called (minus) semisimple continued fractions denote by $\left\langle\left\langle a_{0} ; a_{1}, \ldots, a_{n}\right\rangle\right\rangle$ the former and $\left[\left[b_{0} ; b_{1}, \ldots, b_{n}\right]\right]$ the latter respectively. We also define

$$
\left\langle\left\langle a_{0} ; a_{1}\right\rangle\right\rangle=\left[\left[a_{0} ; a_{1}\right]\right]=\frac{a_{0}}{a_{1}} .
$$

When every $a_{i}$ are Lucas [resp. Fibonacci] numbers, the semisimple continued fraction is called Lucas [resp. Fibonacci] continued fraction. For instance, $\frac{L_{22}}{L_{17}}$ equals the Lucas continued fraction $\left\langle\left\langle L_{5} ; L_{5}, L_{5}, L_{7}, L_{2}\right\rangle\right\rangle$. This provides a good reason to define the semisimple continued fraction.

We may compare these fractions to the (minus) simple continued fractions

$$
a_{0}+\frac{1}{a_{1}+\frac{1}{\ddots \frac{1}{a_{n-1}+\frac{1}{a_{n}}}}} \text { and } b_{0}-\frac{1}{b_{1}-\frac{1}{\ddots \frac{1}{b_{n-1}-\frac{1}{b_{n}}}}} \text {, }
$$

where $a_{0}, b_{0} \geq 0$ and $a_{i}>0, b_{i}>1(i=1, \ldots, n)$, denoted by $\left\langle a_{0} ; a_{1}, \ldots, a_{n}\right\rangle$ and $\left[b_{0} ; b_{1}, \ldots, b_{n}\right]$, respectively (refer to $[1]$ ).

Theorem 2.2. Let $n \geq 2$. For the (minus) semisimple continued fractions,

(1)

$$
\begin{aligned}
\left\langle\left\langle a_{0} ; \ldots, a_{n}\right\rangle\right\rangle & =a_{0}+\left\langle\left\langle 0 ; a_{1}, \ldots, a_{n}\right\rangle\right\rangle \\
& =a_{0}+\frac{1}{\left\langle\left\langle a_{1} ; \ldots, a_{n}\right\rangle\right\rangle}
\end{aligned}
$$


(2)

$$
\begin{aligned}
& =\frac{1}{\left\langle\left\langle 0 ; a_{0}, \ldots, a_{n}\right\rangle\right\rangle} \\
& =\left\langle\left\langle a_{0} ; \ldots, a_{n-3}, a_{n-2} a_{n-1}+a_{n}, a_{n-1}\right\rangle\right\rangle \\
& =\left\langle\left\langle a_{0} ; \ldots, a_{n-2}, \frac{a_{n-2} a_{n-1}}{a_{n-2} a_{n-1}+a_{n}}\right\rangle\right\rangle .
\end{aligned}
$$

$$
\begin{aligned}
{\left[\left[a_{0} ; \ldots, a_{n}\right]\right] } & =a_{0}+\left[\left[0 ; a_{1}, \ldots, a_{n}\right]\right] \\
& =a_{0}-\frac{1}{\left[\left[a_{1} ; \ldots, a_{n}\right]\right]} \\
& =-\frac{1}{\left[\left[0 ; a_{0}, \ldots, a_{n}\right]\right]} \\
& =\left\langle\left\langle a_{0} ; \ldots, a_{n-2},\left\lfloor\frac{a_{n-1}}{a_{n}}\right\rfloor, a_{n}, a_{n-1}-\left\lfloor\frac{a_{n-1}}{a_{n}}\right\rfloor a_{n}\right\rangle\right\rangle .
\end{aligned}
$$

Proof. Since

$$
\begin{aligned}
\left\langle\left\langle a_{0} ; \ldots, a_{n-2}, a_{n-1}, a_{n}\right\rangle\right\rangle & =a_{0}+\frac{1}{\ddots a_{n-3}+\frac{1}{a_{n-2}+\frac{1}{\frac{a_{n-1}}{a_{n}}}}} \\
& =a_{0}+\frac{1}{\ddots a_{n-3}+\frac{1}{\frac{a_{n-2} a_{n-1}+a_{n}}{a_{n-1}}}} \\
& =a_{0}+\frac{1}{\ddots a_{n-3}+\frac{1}{\frac{a_{n-2}}{x}}}
\end{aligned}
$$

with $x=\frac{a_{n-2} a_{n-1}}{a_{n-2} a_{n-1}+a_{n}}$, (1) follows immediately.

Now for (2), write $a_{n-1}=q a_{n}+r$ with $0 \leq r<a_{n}$ and $q \in \mathbb{Z}$. Then

$$
\begin{aligned}
\left\langle\left\langle a ; a_{n-1}, a_{n}\right\rangle\right\rangle & =a+\frac{1}{\frac{a_{n-1}}{a_{n}}}=a+\frac{1}{q+\frac{1}{\frac{a_{n}}{r}}}=\left\langle\left\langle a ; q, a_{n}, r\right\rangle\right\rangle \\
& =\left\langle\left\langle a ;\left\lfloor\frac{a_{n-1}}{a_{n}}\right\rfloor, a_{n}, a_{n-1}-\left\lfloor\frac{a_{n-1}}{a_{n}}\right\rfloor a_{n}\right\rangle\right\rangle .
\end{aligned}
$$

Theorem 2.3. We further have the following identities.

(1) $\left\langle\left\langle a_{0} ; \ldots, a_{n}, 1\right\rangle\right\rangle=\left\langle a_{0} ; \ldots, a_{n}\right\rangle,\left[\left[a_{0} ; \ldots, a_{n}, 1\right]\right]=\left[a_{0} ; \ldots, a_{n}\right]$ for $n \geq$ 1.

(2) $\left\langle\left\langle a_{0} ; \ldots, a_{n}\right\rangle\right\rangle=\left\langle a_{0} ; \ldots, a_{n-2}, \frac{a_{n-1}}{a_{n}}\right\rangle=\left\langle a_{0} ; \ldots, a_{n-3}, a_{n-2}+\frac{a_{n}}{a_{n-1}}\right\rangle$ for $n \geq 3$.

(3) $\left\langle\left\langle a_{0} ; a_{1}, \ldots, a_{n}\right\rangle\right\rangle=\left\langle a_{0} ; a_{1}, \ldots, a_{k},\left\langle\left\langle a_{k+1} ; \ldots, a_{n}\right\rangle\right\rangle\right\rangle$ for $0 \leq k \leq n-2$. 
Proof. The proof is not hard.

Example. $\langle\langle 1 ; 2,3,4,5,6\rangle\rangle=\langle\langle 1 ; 2,3,26,5\rangle\rangle=\langle\langle 1 ; 2,83,26\rangle\rangle=\langle\langle 1 ; 192,83\rangle\rangle$ $=\frac{275}{192}$ by Theorems 2.2 and 2.3 . On the other hand, $\langle\langle 1 ; 2,3,4,5,6\rangle\rangle$ is also equal to $\left\langle\left\langle 1 ; 2,3,4, \frac{10}{13}\right\rangle\right\rangle=\left\langle\left\langle 1 ; 2,3, \frac{78}{83}\right\rangle\right\rangle=\left\langle\left\langle 1 ; 2, \frac{83}{96}\right\rangle\right\rangle=\left\langle\left\langle 1 ; \frac{192}{275}\right\rangle\right\rangle=\frac{275}{192}$.

Similar to the usual notation $\left\langle a^{t}, b\right\rangle=\langle\underbrace{a ; \ldots, a}_{t}, b\rangle$, we denote the $t$ times repeated semisimple continued fractions $\langle\langle a ; \ldots, a, b\rangle\rangle$ and $[[a ; \ldots, a, b]]$ by $\left\langle\left\langle a^{t}, b\right\rangle\right\rangle$ and $\left[\left[a^{t}, b\right]\right]$, respectively.

\section{Convergents of a semisimple continued fraction}

The section is devoted to investigating successive convergents of a semisimple continued fraction, and to studying relationships with those of a simple continued fraction.

Given a continued fraction $\left\langle a_{0} ; a_{1}, \ldots, a_{n}\right\rangle$, a numerator $u_{k}$ and a denominator $v_{k}$ of the $k$ th convergent $C_{k}$ are given by the recursive formulas

$$
u_{k}=u_{k-1} a_{k}+u_{k-2} \text { and } v_{k}=v_{k-1} a_{k}+v_{k-2} \quad(k \geq 1) \text {, }
$$

where $u_{-1}=1, u_{0}=a_{0}$ and $v_{-1}=0, v_{0}=1$. Then (refer to [1])

$$
C_{k}=\left\langle a_{0} ; a_{1}, \ldots, a_{k}\right\rangle=\frac{u_{k}}{v_{k}} \text { and } u_{k} v_{k-1}-u_{k-1} v_{k}=(-1)^{k+1} .
$$

The next theorem shows a recursion formula involving the semisimple continued fraction. For every $a_{k}>0$, let

$$
S_{n}=\left\langle\left\langle a_{0} ; a_{1}, \ldots, a_{n}\right\rangle\right\rangle .
$$

Theorem 3.1. Let $n \geq 2$. Then for all $2 \leq k \leq n$, the following are equivalent for the kth convergent $S_{k}$ of $S_{n}$.

(1) $S_{k}=\left\langle\left\langle a_{0} ; a_{1}, \ldots, a_{k}\right\rangle\right\rangle=\frac{p_{k}}{q_{k}}$.

(2) $p_{k}=u_{k-2} a_{k-1}+u_{k-3} a_{k}$ and $q_{k}=v_{k-2} a_{k-1}+v_{k-3} a_{k}$.

$$
\begin{aligned}
& {\left[\begin{array}{cr}
a_{0} & 1 \\
1 & 0
\end{array}\right]\left[\begin{array}{cc}
a_{1} & 1 \\
1 & 0
\end{array}\right] \ldots\left[\begin{array}{cc}
a_{k-2} & 1 \\
1 & 0
\end{array}\right]\left[\begin{array}{cc}
a_{k-1} & a_{k-1} \\
a_{n} & a_{k-2}-a_{k-2} a_{k-1}
\end{array}\right] } \\
= & {\left[\begin{array}{ll}
u_{k-2} & u_{k-3} \\
v_{k-2} & v_{k-3}
\end{array}\right]\left[\begin{array}{cc}
a_{k-1} & a_{k-1} \\
a_{k} & a_{k-2}-a_{k-2} a_{k-1}
\end{array}\right]=\left[\begin{array}{cc}
p_{k} & p_{k-1} \\
q_{k} & q_{k-1}
\end{array}\right] . }
\end{aligned}
$$

Proof. If $k=2$, then

$$
S_{2}=\left\langle\left\langle a_{0} ; a_{1}, a_{2}\right\rangle\right\rangle=\frac{a_{0} a_{1}+a_{2}}{a_{1}}=\frac{p_{2}}{q_{2}}
$$

implies $p_{2}=a_{0} a_{1}+a_{2}=u_{0} a_{1}+u_{-1} a_{2}$ and $q_{2}=a_{1}=v_{0} a_{1}+v_{-1} a_{2}$. Furthermore

$$
\begin{aligned}
{\left[\begin{array}{cc}
a_{0} & 1 \\
1 & 0
\end{array}\right]\left[\begin{array}{cc}
a_{1} & a_{1} \\
a_{2} & a_{0}-a_{0} a_{1}
\end{array}\right] } & =\left[\begin{array}{cc}
u_{0} & u_{-1} \\
v_{0} & v_{-1}
\end{array}\right]\left[\begin{array}{cc}
a_{1} & a_{1} \\
a_{2} & a_{0}-a_{0} a_{1}
\end{array}\right] \\
& =\left[\begin{array}{cc}
a_{0} a_{1}+a_{2} & a_{0} \\
a_{1} & a_{1}
\end{array}\right]=\left[\begin{array}{cc}
p_{2} & p_{1} \\
q_{2} & q_{1}
\end{array}\right] .
\end{aligned}
$$


On the other hand, Theorem 2.3(2) together with the consideration about the continued fraction give rise to

$$
\begin{aligned}
S_{2} & =\left\langle\left\langle a_{0} ; a_{1}, a_{2}\right\rangle\right\rangle=\left\langle a_{0} ; \frac{a_{1}}{a_{2}}\right\rangle=\frac{u_{0} \frac{a_{1}}{a_{2}}+u_{-1}}{v_{0} \frac{a_{1}}{a_{2}}+v_{-1}} \\
& =\frac{\left(a_{0} a_{1}+a_{2}\right) / a_{2}}{a_{1} / a_{2}}=\frac{a_{0} a_{1}+a_{2}}{a_{1}}=\frac{p_{2}}{q_{2}} .
\end{aligned}
$$

We now suppose that (1), (2) and (3) hold for $S_{k}$ for $k<n$. Then

$$
\begin{aligned}
S_{k} & =\left\langle\left\langle a_{0} ; a_{1}, \ldots, a_{k-1}, a_{k}\right\rangle\right\rangle=\left\langle a_{0} ; a_{1}, \ldots, a_{k-2}, \frac{a_{k-1}}{a_{k}}\right\rangle \\
& =\frac{u_{k-1}}{v_{k-1}}=\frac{u_{k-2} \frac{a_{k-1}}{a_{k}}+u_{k-3}}{v_{k-2} \frac{a_{k-1}}{a_{k}}+v_{k-3}} \\
& =\frac{u_{k-2} a_{k-1}+u_{k-3} a_{k}}{v_{k-2} a_{k-1}+v_{k-3} a_{k}}=\frac{p_{k}}{q_{k}} .
\end{aligned}
$$

Moreover it follows inductively that

$$
\begin{aligned}
& {\left[\begin{array}{ll}
a_{0} & 1 \\
1 & 0
\end{array}\right] \ldots\left[\begin{array}{cc}
a_{k-3} & 1 \\
1 & 0
\end{array}\right]\left[\begin{array}{cc}
a_{k-2} & 1 \\
1 & 0
\end{array}\right]\left[\begin{array}{cc}
a_{k-1} & a_{k-1} \\
a_{k} & a_{k-2}-a_{k-2} a_{k-1}
\end{array}\right] } \\
= & {\left[\begin{array}{ll}
u_{k-3} a_{k-2}+u_{k-4} & u_{k-3} \\
v_{k-3} a_{k-2}+v_{k-4} & v_{k-3}
\end{array}\right]\left[\begin{array}{cc}
a_{k-1} & a_{k-1} \\
a_{k} & a_{k-2}-a_{k-2} a_{k-1}
\end{array}\right] } \\
= & {\left[\begin{array}{ll}
u_{k-2} & u_{k-3} \\
v_{k-2} & v_{k-3}
\end{array}\right]\left[\begin{array}{cc}
a_{k-1} & a_{k-1} \\
a_{k} & a_{k-2}-a_{k-2} a_{k-1}
\end{array}\right]=\left[\begin{array}{cc}
p_{k} & p_{k-1} \\
q_{k} & q_{k-1}
\end{array}\right] }
\end{aligned}
$$

if and only if $p_{k}=u_{k-2} a_{k-1}+u_{k-3} a_{k}$ and $q_{k}=v_{k-2} a_{k-1}+v_{k-3} k_{n}$.

Example. Consider $\frac{p_{5}}{q_{5}}=\langle\langle 1 ; 2,3,4,5,6\rangle\rangle$. Since $\langle 1 ; 2,3\rangle=\frac{10}{7}=\frac{u_{2}}{v_{2}}$ and $\langle 1 ; 2,3,4\rangle=\frac{43}{30}=\frac{u_{3}}{v_{3}},\left[\begin{array}{c}p_{5} \\ q_{5}\end{array}\right]=\left[\begin{array}{cc}40 & 10 \\ 30 & 7\end{array}\right]\left[\begin{array}{l}5 \\ 6\end{array}\right]=\left[\begin{array}{c}275 \\ 192\end{array}\right]$ so $\langle\langle 1 ; 2,3,4,5,6\rangle\rangle=\frac{275}{192}$.

From now on, we denote the semisimple continued fraction $\left\langle\left\langle a_{0} ; a_{1}, \ldots, a_{n}\right\rangle\right\rangle$ by $S_{n}$ and the continued fraction $\left\langle a_{0} ; a_{1}, \ldots, a_{n}\right\rangle$ by $C_{n}$ where $0<a_{i} \in \mathbb{Z}$. Then for every $k<n, S_{k}=\frac{p_{k}}{q_{k}}$ and $C_{k}=\frac{u_{k}}{v_{k}}$ are the $k$ th convergents of $S_{n}$ and $C_{n}$ respectively, where $u_{k}, v_{k}, p_{k}$ and $q_{k}$ are in Theorem 3.1.

Lemma 3.2. $C_{k}-C_{k-1}=\frac{(-1)^{k+1}}{v_{k-1} v_{k}}$ and $C_{k}-C_{k-2}=\frac{(-1)^{k+1}}{v_{k-2} v_{k}} a_{k}$ for $k \geq 1$.

Proof. It is clear to see that

$$
C_{k}-C_{k-1}=\frac{u_{k}}{v_{k}}-\frac{u_{k-1}}{v_{k-1}}=\frac{u_{k} v_{k-1}-u_{k-1} v_{k}}{v_{k-1} v_{k}}=\frac{(-1)^{k+1}}{v_{k-1} v_{k}} .
$$

Furthermore, since $v_{k}=v_{k-1} a_{k}+v_{k-2}$ we have

$$
C_{k}-C_{k-2}=\frac{(-1)^{k+1}}{v_{k-1} v_{k}}+\frac{(-1)^{k}}{v_{k-1} v_{k-2}}=\frac{(-1)^{k} v_{k-1} a_{k}}{v_{k-2} v_{k-1} v_{k}}=\frac{(-1)^{k} a_{k}}{v_{k-2} v_{k}} \text {. }
$$


It shows $C_{0}<C_{2}<C_{4}<\cdots<C_{5}<C_{3}<C_{1}$ and $\left\{C_{k}\right\}$ converges to $C_{n}$ for large enough $n([1])$. Similarly, we shall investigate the differences $S_{k}-S_{k-1}$ and $S_{k}-S_{k-2}$.

Theorem 3.3. Let $\delta_{k}=a_{k-1} a_{k}-a_{k-1}+a_{k+1}$. Then for $k \geq 1$,

(1) $S_{k}-S_{k-1}=\frac{(-1)^{k}}{q_{k-1} q_{k}} a_{k-1} \delta_{k-1}$.

(2) $S_{k}-S_{k-2}=\frac{(-1)^{k}}{q_{k-2} q_{k}}\left(a_{k-1} \delta_{k-1}-a_{k-2} \delta_{k-2}-\delta_{k-2} \delta_{k-1}\right)$.

Proof. By Theorem 3.1, we have

$$
\left[\begin{array}{cc}
u_{k-2} & u_{k-3} \\
v_{k-2} & v_{k-3}
\end{array}\right]\left[\begin{array}{cc}
a_{k-1} & a_{k-1} \\
a_{k} & a_{k-2}-a_{k-2} a_{k-1}
\end{array}\right]=\left[\begin{array}{cc}
p_{k} & p_{k-1} \\
q_{k} & q_{k-1}
\end{array}\right] .
$$

Then the determinants of both sides yield

$$
p_{k} q_{k-1}-p_{k-1} q_{k}=(-1)^{k} a_{k-1}\left(a_{k-2} a_{k-1}-a_{k-2}+a_{k}\right) .
$$

Now by setting $\delta_{k-1}=a_{k-2} a_{k-1}-a_{k-2}+a_{k}$, we have

$$
S_{k}-S_{k-1}=\frac{p_{k}}{q_{k}}-\frac{p_{k-1}}{q_{k-1}}=\frac{p_{k} q_{k-1}-p_{k-1} q_{k}}{q_{k-1} q_{k}}=\frac{(-1)^{k} a_{k-1} \delta_{k-1}}{q_{k-1} q_{k}} .
$$

Again

$$
\begin{aligned}
S_{k}-S_{k-2} & =\frac{(-1)^{k} a_{k-1} \delta_{k-1}}{q_{k-1} q_{k}}+\frac{(-1)^{k-1} a_{k-2} \delta_{k-2}}{q_{k-2} q_{k-1}} \\
& =\frac{(-1)^{k}}{q_{k-2} q_{k-1} q_{k}}\left(a_{k-1} \delta_{k-1} q_{k-2}-a_{k-2} \delta_{k-2} q_{k}\right) .
\end{aligned}
$$

From the identities $v_{k}=v_{k-1} a_{k}+v_{k-2}$ and $q_{k}=v_{k-2} a_{k-1}+v_{k-3} a_{k}$ in Theorem 3.1 , we have

$$
\begin{aligned}
q_{k} & =\left(v_{k-3} a_{k-2}+v_{k-4}\right) a_{k-1}+v_{k-3} a_{k} \\
& =v_{k-3}\left(a_{k-2} a_{k-1}+a_{k}\right)+v_{k-4} a_{k-1} \\
& =v_{k-3}\left(a_{k-2} a_{k-1}+a_{k}\right)+\left(q_{k-1}-v_{k-3} a_{k-2}\right) \\
& =v_{k-3}\left(a_{k-2} a_{k-1}-a_{k-2}+a_{k}\right)+q_{k-1}=v_{k-3} \delta_{k-1}+q_{k-1} .
\end{aligned}
$$

Thus

$$
\begin{aligned}
& a_{k-1} \delta_{k-1} q_{k-2}-a_{k-2} \delta_{k-2} q_{k} \\
= & q_{k-1}\left(a_{k-1} \delta_{k-1}-a_{k-2} \delta_{k-2}\right)-\delta_{k-2} \delta_{k-1}\left(v_{k-3} a_{k-2}+v_{k-4} a_{k-1}\right) \\
= & q_{k-1}\left(a_{k-1} \delta_{k-1}-a_{k-2} \delta_{k-2}\right)-\delta_{k-2} \delta_{k-1} q_{k-1} \\
= & q_{k-1}\left(a_{k-1} \delta_{k-1}-a_{k-2} \delta_{k-2}-\delta_{k-2} \delta_{k-1}\right),
\end{aligned}
$$

so

$$
\begin{aligned}
S_{k}-S_{k-2} & =\frac{(-1)^{k}}{q_{k-2} q_{k-1} q_{k}} q_{k-1}\left(a_{k-1} \delta_{k-1}-a_{k-2} \delta_{k-2}-\delta_{k-2} \delta_{k-1}\right) \\
& =\frac{(-1)^{k}}{q_{k-2} q_{k}}\left(a_{k-1} \delta_{k-1}-a_{k-2} \delta_{k-2}-\delta_{k-2} \delta_{k-1}\right) .
\end{aligned}
$$


Theorem 3.4. $S_{3}<S_{5}<S_{7}<\cdots<S_{6}<S_{4}<S_{2}$, so $\left\{S_{k}\right\}$ converges to $S_{n}$ for large enough $n$.

Proof. Obviously $q_{k}>0$ and $\delta_{k}=a_{k-1}\left(a_{k}-1\right)+a_{k+1}>0$ for every $k$. Thus $S_{k}-S_{k-1}=\frac{(-1)^{k}}{q_{k-1} q_{k}} a_{k-1} \delta_{k}$ shows $S_{k-1}<S_{k}$ if $k$ is even, otherwise $S_{k}<S_{k-1}$. Furthermore we have

$$
\begin{aligned}
& a_{k-1} \delta_{k-1}-a_{k-2} \delta_{k-2}-\delta_{k-2} \delta_{k-1} \\
= & -a_{k-2} a_{k-1}-a_{k-3} a_{k-2}^{2} a_{k-1}+a_{k-3} a_{k-2} a_{k-1}-a_{k-3} a_{k-2} a_{k}+a_{k-3} a_{k} \\
= & -a_{k-2} a_{k-1}\left(1+a_{k-3}\left(a_{k-2}-1\right)\right)-a_{k-3} a_{k}\left(a_{k-2}-1\right) \\
= & -\left(a_{k-3}\left(a_{k-2}-1\right)\left(a_{k-2} a_{k-1}+a_{k}\right)+a_{k-2} a_{k-1}\right)<0 .
\end{aligned}
$$

Thus from

$$
S_{k}-S_{k-2}=\frac{(-1)^{k+1}}{q_{k} q_{k-2}}\left(a_{k-3}\left(a_{k-2}-1\right)\left(a_{k-2} a_{k-1}+a_{k}\right)+a_{k-2} a_{k-1}\right),
$$

we have $S_{k}<S_{k-2}$ for even $k$, otherwise $S_{k-2}<S_{k}$. Therefore

$$
S_{3}<S_{5}<S_{7}<\cdots<S_{6}<S_{4}<S_{2},
$$

so $S_{k}$ converges when $k$ gets larger.

For example, from $\pi=\left\langle a_{0} ; a_{1}, a_{2}, \ldots\right\rangle=\langle 3 ; 7,15,1,292,1,1,1,2,1,3,1,14$, $\ldots\rangle$, the first few convergents of continued fraction are

$$
C_{0}=\langle 3\rangle=3=\frac{u_{0}}{v_{0}}, \quad C_{1}=\langle 3 ; 7\rangle=\frac{22}{7}=\frac{u_{1}}{v_{1}} .
$$

Moreover

$$
C_{2}=\langle 3 ; 7,15\rangle=\frac{u_{1} a_{2}+u_{0}}{v_{1} a_{2}+v_{0}}=\frac{22 \cdot 15+3}{7 \cdot 15+1}=\frac{333}{106}=\frac{u_{2}}{v_{2}},
$$

and similarly

$$
C_{3}=\langle 3 ; 7,15,1\rangle=\frac{355}{113}=\frac{u_{3}}{v_{3}}, C_{4}=\frac{103993}{33102}, C_{5}=\frac{104348}{33215}, \ldots
$$

Let us consider $S=\left\langle\left\langle a_{0} ; a_{1}, \ldots\right\rangle\right\rangle=\langle\langle 3 ; 7,15,1,292,1,1,1,2, \ldots\rangle\rangle$. Then

$$
S_{1}=\langle\langle 3 ; 7\rangle\rangle=\frac{3}{7}=\frac{p_{1}}{q_{1}} \text { and } S_{2}=\langle\langle 3 ; 7,15\rangle\rangle=\frac{36}{7}=\frac{p_{2}}{q_{2}} .
$$

And Theorem 3.1 shows that

$$
S_{3}=\langle\langle 3 ; 7,15,1\rangle\rangle=\frac{p_{3}}{q_{3}}=\frac{u_{1} a_{2}+u_{0} a_{3}}{v_{1} a_{2}+v_{0} a_{3}}=\frac{22 \cdot 15+3 \cdot 1}{7 \cdot 15+1 \cdot 1}=\frac{333}{106}
$$

and

$$
S_{4}=\frac{333 \cdot 1+22 \cdot 292}{106 \cdot 1+7 \cdot 292}=\frac{6757}{2150}, S_{5}=\frac{355 \cdot 292+333}{113 \cdot 292+106}=\frac{103993}{33102}, \ldots
$$

Now the differences of convergents of $C_{n}$ are, for instance

$C_{4}-C_{3}=\frac{-1}{(113)(33102)}, C_{5}-C_{4}=\frac{1}{(33102)(33215)}$ and $C_{4}-C_{2}=\frac{292}{(106)(33102)}$. 
Moreover Theorem 3.3 shows

and

$$
S_{5}-S_{4}=\frac{-292(292+1-1)}{2150 \cdot 33102}=\frac{-292^{2}}{71169300}
$$

$$
S_{6}-S_{4}=\frac{-1}{2150 \cdot 33215}((292+1)(292-1)+292)=\frac{85555}{71412250} .
$$

Corollary 3.5. Let $S_{n}=\left\langle\left\langle a_{0} ; a_{1}, \ldots, a_{n}\right\rangle\right\rangle=\frac{p_{n}}{q_{n}}$. Assume $a_{k}=1$ for $k<n$. Then $S_{k+1}-S_{k}=\frac{(-1)^{k+1} a_{k+1}}{q_{k} q_{k+1}}$ and $S_{k+2}-S_{k}=\frac{(-1)^{k+1} a_{k+1}}{q_{k} q_{k+2}}$.

Proof. From Theorem 3.3, we have

$$
S_{k+1}-S_{k}=\frac{(-1)^{k+1}}{q_{k} q_{k+1}}\left(a_{k}\left(a_{k-1}\left(a_{k}-1\right)+a_{k+1}\right)\right)=\frac{(-1)^{k+1} a_{k+1}}{q_{k} q_{k+1}} .
$$

Similarly,

$$
\begin{aligned}
S_{k+2}-S_{k} & =\frac{(-1)^{k+1}}{q_{k} q_{k+2}}\left(a_{k-1}\left(a_{k}-1\right)\left(a_{k} a_{k+1}+a_{k+2}\right)+a_{k} a_{k+1}\right) \\
& =\frac{(-1)^{k+1} a_{k+1}}{q_{k} q_{k+2}} .
\end{aligned}
$$

From $S=\langle\langle 3 ; 7,15,1,292,1,1, \ldots\rangle\rangle$ in the above example, since $a_{3}=1$ and $a_{4}=292$, we have $S_{4}-S_{3}=\frac{292}{q_{3} q_{4}}$ and $S_{5}-S_{3}=\frac{292}{q_{3} q_{5}}$.

\section{Ratios of Fibonacci numbers in semisimple continued fraction}

It is well known that the ratio $\frac{F_{n+1}}{F_{n}}$ is equal to $\left\langle 1^{n}\right\rangle=\langle 1 ; 1, \ldots, 1\rangle$. This section is devoted to study $\frac{F_{n}}{F_{k}}$ for any $n$ and $k$. The fractions $\langle\cdots\rangle,[\cdots]$, $\langle\langle\cdots\rangle\rangle$ or $[[\cdots]]$ will be put together to express the ratios effectively.

Lemma 4.1. Let $n \geq 3$. Then $F_{n+1}=2 F_{n}-F_{n-2}=2 F_{n-1}+F_{n-2}$ and $F_{n+2}=3 F_{n}-F_{n-2}=L_{2} F_{n}-F_{n-2}$.

Proof. Obviously $F_{n+1}=F_{n}+F_{n-1}=F_{n}+\left(F_{n}-F_{n-2}\right)=2 F_{n}-F_{n-2}$, while $F_{n+1}=F_{n}+F_{n-1}=\left(F_{n-1}+F_{n-2}\right)+F_{n-1}=2 F_{n-1}+F_{n-2}$. And $F_{n+2}=F_{n+1}+F_{n}=\left(2 F_{n}-F_{n-2}\right)+F_{n}=3 F_{n}-F_{n-2}$.

Moreover due to Lemma 2.1, $F_{n+k}=L_{k} F_{n}+(-1)^{k-1} F_{n-k}$, so we have $F_{n+2}=L_{2} F_{n}-F_{n-2}$ if $k=2$.

Theorem 4.2. Let $n \geq 3$ and write $n=2 t+r$ with $1 \leq r \leq 2$.
(1) $\frac{F_{n+2}}{F_{n}}=\left\langle 2 ; 1^{n-1}\right\rangle= \begin{cases}{\left[\left[3^{t+1}, 1\right]\right]=\left[3^{t+1}\right]} & \text { if } r=2, \\ {\left[\left[3^{t}, 2,1\right]\right]=\left[3^{t}, 2\right]} & \text { if } r=1 .\end{cases}$
(2) $\frac{F_{n+1}}{F_{n}}=\left\langle 1^{n}\right\rangle= \begin{cases}{\left[\left[2 ; 3^{t}, 1\right]\right]} & \text { if } r=2, \\ {\left[\left[2 ; 3^{t-1}, 2,1\right]\right]} & \text { if } r=1 .\end{cases}$ 
Proof. Due to Lemma 4.1, it is obvious that

$$
\frac{F_{n+2}}{F_{n}}=\frac{2 F_{n}+F_{n-1}}{F_{n}}=2+\frac{1}{\frac{F_{n}}{F_{n-1}}}=2+\frac{1}{\left\langle 1^{n-1}\right\rangle}=\left\langle 2 ; 1^{n-1}\right\rangle \text {, }
$$

and

$$
\begin{aligned}
\frac{F_{n+2}}{F_{n}} & =\frac{3 F_{n}-F_{n-2}}{F_{n}}=3-\frac{1}{\frac{F_{n}}{F_{n-2}}}=3-\frac{1}{\frac{3 F_{n-2}-F_{n-4}}{F_{n-2}}}=3-\frac{1}{3-\frac{1}{\frac{F_{n-2}}{F_{n-4}}}} \\
& \left.=\cdots=3-\frac{1}{3-\frac{1}{\ddots 3-\frac{F_{n-2(v-1)}}{F_{n-2 v}}}}=[\underbrace{3 ; \ldots, 3}_{v}, F_{n-2(v-1)}, F_{n-2 v}]\right]
\end{aligned}
$$

for $v \leq t$. Therefore, for $n=2 t+r(1 \leq r \leq 2)$, Theorem 2.3 yields

$$
\begin{aligned}
\frac{F_{n+2}}{F_{n}} & =\left[\left[3^{t}, F_{2+r}, F_{r}\right]\right] \\
& = \begin{cases}{\left[\left[3^{t}, F_{4}, F_{2}\right]\right]=\left[\left[3^{t}, 3,1\right]\right]=\left[3^{t+1}\right]} & \text { if } r=2, \\
{\left[\left[3^{t}, F_{3}, F_{1}\right]\right]=\left[\left[3^{t}, 2,1\right]\right]=\left[3^{t}, 2\right]} & \text { if } r=1 .\end{cases}
\end{aligned}
$$

On the other hand, since $2 F_{n}-F_{n-2}=F_{n+1}$, it follows from (1) that

$$
\begin{aligned}
\frac{F_{n+1}}{F_{n}} & =\frac{2 F_{n}-F_{n-2}}{F_{n}}=2-\frac{1}{\frac{F_{n}}{F_{n-2}}} \\
& = \begin{cases}2-\frac{1}{\left[\left[3^{t}, 1\right]\right]}=\left[\left[2 ; 3^{t}, 1\right]\right] & \text { if } r=2, \\
2-\frac{1}{\left[\left[3^{t-1}, 2,1\right]\right]}=\left[\left[2 ; 3^{t-1}, 2,1\right]\right] & \text { if } r=1 .\end{cases}
\end{aligned}
$$

It shows that if $n=2 t+r(r=1,2)$, then $\frac{F_{n+2}}{F_{n}}=\left\langle 2 ; 1^{n-1}\right\rangle$ equals either $\left[\left[3^{t+1}, 1\right]\right]$ or $\left[\left[3^{t}, 2,1\right]\right]$, and $\frac{F_{n+1}}{F_{n}}=\left\langle 1^{n}\right\rangle$ equals either $\left[\left[2 ; 3^{t}, 1\right]\right]$ or $\left[\left[2 ; 3^{t-1}, 2,1\right]\right]$. This means that while $n$ repeated computations are needed in the simple continued fraction, only about $t=\left\lfloor\frac{n}{2}\right\rfloor$ repeated computations are required in the semisimple continued fractions. For example, if $n=6$, then $[[2 ; 3,3]]=\frac{F_{7}}{F_{6}}=$ $\langle 1 ; 1,1,1,1,1\rangle$. Hence if $n$ is large, a semisimple continued fraction is more convenient than a simple continued fraction.

Theorem 4.3. Let $n \geq 4$ and write $n=3 t+r$ with $1 \leq r \leq 3$. Then $\frac{F_{n+3}}{F_{n}}=$ $\left\langle\left\langle L_{3}^{t}, F_{3+r}, F_{r}\right\rangle\right\rangle$, which is equal to one of $\left\langle 4^{t}, 3\right\rangle,\left\langle 4^{t}, 5\right\rangle$ or $\left\langle 4^{t}, 4\right\rangle$ according to $r=1,2,3$.

Proof. Lemma 2.1 with $k=3$ implies $F_{n+3}=L_{3} F_{n}+F_{n-3}$, thus

$$
\frac{F_{n+3}}{F_{n}}=L_{3}+\frac{1}{\frac{F_{n}}{F_{n-3}}}=4+\frac{1}{4+\frac{1}{\frac{F_{n-3}}{F_{n-6}}}}=4+\frac{1}{4+\frac{1}{\ddots 4+\frac{1}{\frac{F_{n-3(t-1)}}{F_{n-3 t}}}}}
$$




$$
=\left\langle\left\langle L_{3}^{t}, F_{n-3(t-1)}, F_{n-3 t}\right\rangle\right\rangle=\left\langle\left\langle 4^{t}, F_{3+r}, F_{r}\right\rangle\right\rangle .
$$

Hence by making use of Theorem 2.3, it follows that

$$
\frac{F_{n+3}}{F_{n}}= \begin{cases}\left\langle\left\langle 4^{t}, 3,1\right\rangle\right\rangle=\left\langle 4^{t}, 3\right\rangle & \text { if } r=1 \\ \left\langle\left\langle 4^{t}, 5,1\right\rangle\right\rangle=\left\langle 4^{t}, 5\right\rangle & \text { if } r=2 \\ \left\langle\left\langle 4^{t}, 8,2\right\rangle\right\rangle=\left\langle 4^{t}, 4\right\rangle & \text { if } r=3\end{cases}
$$

Now we generalize Theorem 4.3 as follows.

Theorem 4.4. Let $k \geq 3$. Let $n \geq k+1$ and write $n=k t+r$ with $1 \leq r \leq k$. Then $\frac{F_{n+k}}{F_{n}}$ equals $\left\langle\left\langle L_{k}^{t}, F_{k+r}, F_{r}\right\rangle\right\rangle$ if $k$ is odd, and $\left[\left[L_{k}^{t}, F_{k+r}, F_{r}\right]\right]$ if $k$ is even. So

$$
\frac{F_{n+k}}{F_{n}}= \begin{cases}\left\langle L_{k}^{t}, F_{k+1}\right\rangle \text { or }\left[L_{k}^{t}, F_{k+1}\right] & \text { if } r=1, \\ \left\langle L_{k}^{t}, F_{k+2}\right\rangle \text { or }\left[L_{k}^{t}, F_{k+2}\right] & \text { if } r=2, \\ \left\langle L_{k}^{t+1}\right\rangle \text { or }\left[L_{k}^{t+1}\right] & \text { if } r=k .\end{cases}
$$

Proof. Let $k$ be odd. Since $F_{n+k}=L_{k} F_{n}+F_{n-k}$ in Lemma 2.1, we have

$$
\frac{F_{n+k}}{F_{n}}=L_{k}+\frac{1}{L_{k}+\frac{1}{\ddots L_{k}+\frac{1}{\frac{F_{k+r}}{F_{r}}}}}=\left\langle\left\langle L_{k}^{t}, F_{k+r}, F_{r}\right\rangle\right\rangle .
$$

If $r=1$ or 2 , then $\frac{F_{n+k}}{F_{n}}$ equals $\left\langle\left\langle L_{k}^{t}, F_{k+1}, 1\right\rangle\right\rangle=\left\langle L_{k}^{t}, F_{k+1}\right\rangle$ or $\left\langle\left\langle L_{k}^{t}, F_{k+2}, 1\right\rangle\right\rangle$ $=\left\langle L_{k}^{t}, F_{k+2}\right\rangle$. If $r=k$, then since $F_{2 k}=F_{k} L_{k}$, it follows from Theorem 2.3 that

$$
\frac{F_{n+k}}{F_{n}}=\left\langle\left\langle L_{k}^{t}, F_{2 k}, F_{k}\right\rangle\right\rangle=\left\langle\left\langle L_{k}^{t}, F_{k} L_{k}, F_{k}\right\rangle\right\rangle=\left\langle L_{k}^{t}, L_{k}\right\rangle=\left\langle L_{k}^{t+1}\right\rangle .
$$

On the other hand, if $k$ is even, then $F_{n+k}=L_{k} F_{n}-F_{n-k}$ thus

$$
\frac{F_{n+k}}{F_{n}}=L_{k}-\frac{1}{\frac{F_{n}}{F_{n-k}}}=L_{k}-\frac{1}{L_{k}-\frac{1}{\frac{F_{n-k}}{F_{n-2 k}}}}=\cdots=\left[\left[L_{k}^{t}, F_{k+r}, F_{r}\right]\right],
$$

and the rest follows similarly.

In particular, in case of $n=k t+r$ with $r=3 \leq k$, the next theorem follows.

Theorem 4.5. With the same notations as in Theorem 4.4, let $r=3$ and write $k=3 u+v$ with $1 \leq v \leq 3$. Set $P_{0}=1$ and $P_{u}=4 P_{u-1}+P_{u-2}$. If $k$ is odd, then

$$
\frac{F_{n+k}}{F_{n}}= \begin{cases}\left\langle L_{k}^{t},\left\langle\left\langle 3 P_{u} ; 2\right\rangle\right\rangle\right\rangle \text { with } P_{1}=\langle 4 ; 3\rangle, & \text { if } v=1, \\ \left\langle L_{k}^{t},\left\langle\left\langle 5 P_{u} ; 2\right\rangle\right\rangle\right\rangle \text { with } P_{1}=\langle 4 ; 5\rangle, & \text { if } v=2, \\ \left\langle L_{k}^{t},\left\langle\left\langle 4 P_{u} ; 2\right\rangle\right\rangle\right\rangle \text { with } P_{1}=\langle 4 ; 4\rangle, & \text { if } v=3\end{cases}
$$


If $k$ is even, then $\frac{F_{n+k}}{F_{n}}$ is either $\left[L_{k}^{t},\left\langle\left\langle 3 P_{u} ; 2\right\rangle\right\rangle\right],\left[L_{k}^{t},\left\langle\left\langle 5 P_{u} ; 2\right\rangle\right\rangle\right]$ or $\left[L_{k}^{t},\left\langle\left\langle 4 P_{u} ; 2\right\rangle\right\rangle\right]$.

Proof. Assume $k$ is odd. Theorem 4.4 together with Theorem 2.3 yields

$$
\frac{F_{n+k}}{F_{n}}=\left\langle\left\langle L_{k}^{t}, F_{k+r}, F_{r}\right\rangle\right\rangle=\left\langle L_{k}^{t},\left\langle\left\langle F_{k+3} ; F_{3}\right\rangle\right\rangle\right\rangle .
$$

Since $3 \leq k$, if we write $k=3 u+v$ with $1 \leq v \leq 3$, then we have

$$
\frac{F_{k+3}}{F_{k}}=\frac{F_{3(u+1)+v}}{F_{3 u+v}}=\left\langle\left\langle L_{3}^{u}, F_{v+3}, F_{v}\right\rangle\right\rangle=\left\langle\left\langle 4^{u}, F_{v+3}, F_{v}\right\rangle\right\rangle=\left\langle 4^{u}, \lambda\right\rangle,
$$

where $\lambda=3,5$ or 4 according to $v=1,2$ or 3 by Theorem 4.3.

Let $P_{0}=1$ and $P_{1}=\langle 4 ; \lambda\rangle$. Then we see that

$$
\begin{aligned}
& \left\langle 4^{2}, \lambda\right\rangle=4+\frac{1}{\langle 4 ; \lambda\rangle}=\frac{4 P_{1}+1}{P_{1}}=\frac{P_{2}}{P_{1}}, \text { where } P_{2}=4 P_{1}+P_{0}, \\
& \left\langle 4^{3}, \lambda\right\rangle=4+\frac{1}{\left\langle 4^{2}, \lambda\right\rangle}=\frac{4 P_{2}+P_{1}}{P_{2}}=\frac{P_{3}}{P_{2}}, \text { where } P_{3}=4 P_{2}+P_{1}, \\
& \left\langle 4^{4}, \lambda\right\rangle=4+\frac{1}{\left\langle 4^{3}, \lambda\right\rangle}=\frac{4 P_{3}+P_{2}}{P_{3}}=\frac{P_{4}}{P_{3}}, \text { where } P_{4}=4 P_{3}+P_{2} .
\end{aligned}
$$

Thus we have, for any $0 \leq i \leq u$,

$$
\frac{F_{3(i+1)+v}}{F_{3 i+v}}=\left\langle 4^{i}, \lambda\right\rangle=\frac{P_{i}}{P_{i-1}}, \text { where } P_{i}=4 P_{i-1}+P_{i-2}
$$

with $P_{0}=1$ and $P_{1}=\langle 4 ; \lambda\rangle$. It then follows that

$$
\begin{aligned}
\left\langle\left\langle F_{k+3} ; F_{3}\right\rangle\right\rangle & =\frac{F_{3(u+1)+v}}{F_{3}} \\
& =\frac{F_{3(u+1)+v}}{F_{3 u+v}} \frac{F_{3 u+v}}{F_{3(u-1)+v}} \cdots \frac{F_{3 \cdot 2+v}}{F_{3+v}} \frac{F_{3+v}}{F_{3}} \\
& =\left\langle 4^{u}, \lambda\right\rangle\left\langle 4^{u-1}, \lambda\right\rangle\left\langle 4^{u-2}, \lambda\right\rangle \cdots\langle 4 ; \lambda\rangle\left\langle\left\langle F_{v+3} ; F_{3}\right\rangle\right\rangle \\
& =\frac{P_{u}}{P_{u-1}} \frac{P_{u-1}}{P_{u-2}} \cdots \frac{P_{2}}{P_{1}} \frac{P_{1}}{1}\left\langle\left\langle F_{v+3} ; F_{3}\right\rangle\right\rangle \\
& =P_{u}\left\langle\left\langle F_{v+3} ; F_{3}\right\rangle\right\rangle .
\end{aligned}
$$

If $v=1$, then $\lambda=3$ and $\frac{F_{k+3}}{F_{k}}=\left\langle 4^{u}, 3\right\rangle$ so

$$
\left\langle\left\langle F_{k+3} ; F_{3}\right\rangle\right\rangle=P_{u}\left\langle\left\langle F_{4} ; F_{3}\right\rangle\right\rangle=P_{u}\langle\langle 3 ; 2\rangle\rangle=\left\langle\left\langle 3 P_{u} ; 2\right\rangle\right\rangle,
$$

with $P_{1}=\langle 4 ; 3\rangle$ and $P_{i}=4 P_{i-1}+P_{i-2}$. Thus $\frac{F_{n+k}}{F_{n}}=\left\langle L_{k}^{t},\left\langle\left\langle 3 P_{u} ; 2\right\rangle\right\rangle\right\rangle$.

If $v=2$, then $\lambda=5$ and $\frac{F_{k+3}}{F_{k}}=\left\langle 4^{u}, 5\right\rangle$ so $\frac{F_{n+k}}{F_{n}}=\left\langle L_{k}^{t},\left\langle\left\langle 5 P_{u} ; 2\right\rangle\right\rangle\right\rangle$ with $P_{1}=\langle 4 ; 5\rangle$. Similarly if $v=3$, then $\lambda=4$ so $\frac{F_{n+k}}{F_{n}}=\left\langle L_{k}^{t},\left\langle\left\langle 4 P_{u} ; 2\right\rangle\right\rangle\right\rangle$ with $P_{1}=\langle 4 ; 4\rangle$.

On the other hand, assume that $k$ is even. Then Theorem 4.4 implies

$$
\frac{F_{n+k}}{F_{n}}=\left[\left[L_{k}^{t}, F_{k+r}, F_{r}\right]\right]
$$


which is equal to $\left[L_{k}^{t},\left[\left[F_{k+3} ; F_{3}\right]\right]\right]=\left[L_{k}^{t},\left\langle\left\langle F_{k+3} ; F_{3}\right\rangle\right\rangle\right]$. Hence for $k=3 u+v$ with $v=1,2,3$, the above calculations yield

$$
\left\langle\left\langle F_{k+3}, F_{3}\right\rangle\right\rangle=P_{u}\left\langle\left\langle F_{v+3}, F_{3}\right\rangle\right\rangle=\left\{\begin{array}{lll}
\left\langle\left\langle 3 P_{u} ; 2\right\rangle\right\rangle & \text { with } P_{1}=\langle 4 ; 3\rangle, & \text { if } v=1, \\
\left\langle\left\langle 5 P_{u} ; 2\right\rangle\right\rangle & \text { with } P_{1}=\langle 4 ; 5\rangle, & \text { if } v=2, \\
\left\langle\left\langle 4 P_{u} ; 2\right\rangle\right\rangle & \text { with } P_{1}=\langle 4 ; 4\rangle, & \text { if } v=3 .
\end{array}\right.
$$

Example. In order to find $F_{105}$ and $F_{88}$, let $n=88$ and $k=17$. Then

$$
\frac{F_{105}}{F_{88}}=\frac{F_{17 \cdot 6+3}}{F_{17 \cdot 5+3}}=\left\langle\left\langle L_{17}^{5}, F_{20}, F_{3}\right\rangle\right\rangle=\left\langle L_{17}^{5},\left\langle\left\langle F_{20} ; F_{3}\right\rangle\right\rangle\right\rangle .
$$

Now for $\left\langle\left\langle F_{20} ; F_{3}\right\rangle\right\rangle$, since $17=3 u+v$ with $u=5, v=2$,

$$
\frac{F_{20}}{F_{17}}=\frac{F_{3 \cdot 6+2}}{F_{3 \cdot 5+2}}=\left\langle\left\langle L_{3}^{5}, F_{5}, F_{2}\right\rangle\right\rangle=\left\langle 4^{5}, 5\right\rangle .
$$

Thus by letting $P_{0}=1, P_{1}=\langle 4 ; 5\rangle=\frac{21}{5}$ and $P_{i}=4 P_{i-1}+P_{i-2}(i \leq 5)$, we get $P_{2}=\frac{89}{5}, P_{3}=\frac{377}{5}, P_{4}=\frac{1597}{5}$ and $P_{5}=\frac{6765}{5}$. Hence

$$
\left\langle\left\langle F_{20} ; F_{3}\right\rangle\right\rangle=\left\langle\left\langle P_{5} F_{5} ; F_{3}\right\rangle\right\rangle=\langle\langle 6765 ; 2\rangle\rangle=\frac{6765}{2} \text {. }
$$

Therefore

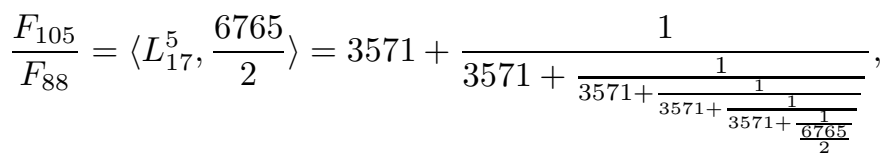

which is $\frac{3,928,413,764,606,871,165,730}{1,100,087,778,366,101,931}$. Here the numerator is $F_{105}$ and the denominator is $F_{88}$, which are 22 digit and 19 digit numbers respectively.

Corollary 4.6. Let $n \geq 6$ and write $n=5 t+r$ with $1 \leq r \leq 5$. Then we have

$$
\frac{F_{n+5}}{F_{n}}=\left\langle\left\langle L_{5}^{t}, F_{5+r}, F_{r}\right\rangle\right\rangle= \begin{cases}\left\langle\left\langle L_{5}^{t}, 8,1\right\rangle\right\rangle=\left\langle 11^{t}, 8\right\rangle & \text { if } r=1, \\ \left\langle\left\langle L_{5}^{t}, 13,1\right\rangle\right\rangle=\left\langle 11^{t}, 13\right\rangle & \text { if } r=2, \\ \left\langle\left\langle L_{5}^{t}, 21,2\right\rangle\right\rangle=\left\langle 11^{t}, 10,2\right\rangle & \text { if } r=3, \\ \left\langle\left\langle L_{5}^{t}, 34,3\right\rangle\right\rangle=\left\langle 11^{t+1}, 3\right\rangle & \text { if } r=4, \\ \left\langle\left\langle L_{5}^{t}, 55,5\right\rangle\right\rangle=\left\langle 11^{t+1}\right\rangle & \text { if } r=5 .\end{cases}
$$

Proof. This is mainly due to Theorem 4.4.

\section{References}

[1] D. Burton, Elementary Number Theory, 3rd ed. WCB, Oxford, England, 1994.

[2] M. Drmota, Fibonacci numbers and continued fraction expansions, in G. E. Vergum et. al (eds.) Applications of Fibonacci numbers, vol 5, 2nd ed. Kluwer Academic Publishers, Netherlands, 1993.

[3] S. Kalia, Fibonacci numbers and continued fractions, MIT PRIMES, 2011. (retrived from web.mit.edu/primes/materials/2011/2011-conf-booklet.pdf) 
[4] S. Katok, Continued fractions, hyperbolic geometry, quadratic froms, in MASS Selecta, Teaching and learning advanced undergraduate mathematics (S. Katok, A. Sossinsky, S. Tabachnikov eds.) American Math. Soc. 2003.

[5] F. Koken and D. Bozkurt, On Lucas numbers by the matrix method, Hacet. J. Math. Stat. 39 (2010), no. 4, 471-475.

[6] T. E. Phipps, Fibonacci and continued fractions, Aperion 15 (2008), no. 4, 534-550.

Department of MATHEMATiCs

HANNAM University

DAEJEON 306-791, KOREA

E-mail address: emc@hnu.kr 